\title{
A Classification of Local Current Algebras
}

\author{
A. JOSEPH \\ Department of Physics and Astronomy Tel-Aviv University, Tel-Aviv, Israel
}

Received May 5, 1970

\begin{abstract}
A simple, yet rigorous derivation of all possible forms of a local Lie algebra $\mathscr{L} g$ subject to a certain finiteness condition is presented. This result is used to describe all possible continuous finite dimensional representations of $\mathscr{L} \mathrm{g}$.
\end{abstract}

\section{Introduction}

The program for the study of the hadrons through current algebra [1-3] presents the problem of finding all possible representations of the so-called local Lie algebras. Here a local Lie algebra $\mathscr{L} g$ is defined to be a Lie algebra (over the real or complex numbers) with generators $V_{\alpha}(\boldsymbol{k})$ : $\alpha=1,2 \ldots \mu ; \boldsymbol{k} \in R^{n}$ (Euclidean $n$-space), satisfying the commutation relations

$$
\left[V_{\alpha}(\boldsymbol{k}), V_{\beta}\left(\boldsymbol{k}^{\prime}\right)\right]=c_{\alpha \beta}^{\gamma} V_{\gamma}\left(\boldsymbol{k}+\boldsymbol{k}^{\prime}\right)
$$

where the $c_{\alpha \beta}^{\gamma}$ denote the structure constants of an arbitrary Lie algebra $g$. (The summation convention is used for the Greek indices throughout). We shall for the most part assume $g$ to be semisimple and finite dimensional and in what follows we refer to $g$ as the Lie algebra on which the local Lie algebra $\mathscr{L} g$ is based. In physical applications of (1.1), the $V_{\alpha}(\boldsymbol{k})$ are identified with the currents expressed in the momentum co-ordinates $\boldsymbol{k}$. The Lie algebra $g$ is supposed to represent an approximate symmetry of the strong interactions, which may for example be $s u(2), s u(3)$ or $s u(3) \times s u(3)$. The larger local algebra $\mathscr{L} g$, in which $g$ is embedded, mixes this internal symmetry with the external space-time co-ordinates and physically may be thought to play the role of a spectrum generating algebra.

Local Lie algebras belong to the class of infinite dimensional Lie algebras and for these a representation theory has not yet been fully developed. In the meantime, Chang, Dashen, and O'Raifeartaigh [4] have suggested studying local Lie algebras whose generators admit an expansion as a finite sum of the form

$$
V_{\alpha}(\boldsymbol{k})=\sum_{r=1}^{m} a_{r}(\boldsymbol{k}) V_{\alpha}^{r}
$$

where the $a_{r}(\boldsymbol{k})$ are real or complex-valued functions carrying the dependence of the momentum variable $\boldsymbol{k}$. In the following we give a rigorous 
derivation of the most general solutions to (1.1) satisfying (1.2). In particular we show that the $V_{\alpha}^{r}$ close on a finite dimensional Lie algebra and obtain the form of this Lie algebra explicitly. This reduces the problem of finding all possible representations of (1.1) satisfying (1.2) to finding all possible representations of a known finite dimensional Lie algebra. This classification embraces all the finite dimensional (unitary and nonunitary) representations of (1.1), since we can easily show that (1.2) is satisfied in this case. In addition an important class of infinite dimensional representations of (1.1) are included in this list.

We begin our analysis in $\S 2$ by taking the $\boldsymbol{k}$-space to be one dimensional $(n=1)$ and $g$ semisimple. This is somewhat simpler than the general problem, yet contains all its general features. In $\S 3$ we extend this argument to all finite $n$. A further generalisation is achieved in $\S 4$ by dropping the requirement that $g$ be semisimple. In $\S 5$ we conclude with a discussion and comparison with previous results.

\section{Classification Theorem $(n=1)$}

In this section we assume the $\boldsymbol{k}$-space to be one dimensional. The analysis we give proceeds by reduction of (1.1) and (1.2) to a Cauchy equation for the $a_{r}(\boldsymbol{k})$. (An excellent account of the Cauchy equation can be found in Aczél [5] which we refer to hereafter as A). To avoid wellknown pathologies (A, p. 35) that can arise in the solution of this equation we impose the following measurability requirement on the $a_{r}(\boldsymbol{k})$, namely: there exists an interval $I \subset R$ (of non-zero measure) such that for all $r=1,2, \ldots m$, the integrals

$$
\int_{I} a_{r}(k) d k \quad \text { exist and are finite. }
$$

A physical justification of (2.1) comes through the need to carry out transformations involving integration of the currents. Given this assumption our main result may be stated as follows.

Theorem 2.1. Let $\mathscr{L} g$ be a local Lie algebra based on the finite dimensional semisimple Lie algebra $g$. Let its generators $V_{\alpha}(k): \alpha=1,2, \ldots \mu$ : $k \in R$ satisfy (1.2) with the measurability requirement (2.1) imposed on the $a_{r}(k): r=1,2 \ldots m$. Then to within a rearrangement of the summation in (1.2) the $V_{\alpha}^{r}$ close on a finite dimensional Lie algebra $h$. Further, there exists a positive integer $j$ such that $h$ is a direct sum of Lie algebras $h_{i}$ : $i=1,2 \ldots j$ of dimension $\mu m_{i}$ with $\sum_{i=1}^{j} m_{i}=m$. Each $h_{i}$ is of the form

$$
\begin{array}{rlrl}
{\left[V_{\alpha}^{r}, V_{\beta}^{s}\right]} & =\frac{(r+s-2) !}{(r-1) !(s-1) !} c_{\alpha \beta}^{\gamma} V_{\gamma}^{r+s-1}: & r+s-1 \leqq m_{i} \\
& =0 \quad: \quad r+s-1>m_{i}
\end{array}
$$

this being a contraction of the $m_{i}$-fold direct sum $g \oplus g \oplus g \oplus \cdots \oplus g$. 
In each $h_{i}, V_{\alpha}(k)$ is given by

$$
V_{\alpha}(k)=\sum_{r=1}^{m_{2}} k^{r-1}(\exp \xi k) V_{\alpha}^{r}
$$

where $\xi$ is an arbitrary constant.

Remark. The first part of this theorem, namely that the $V_{\alpha}^{r}$ close on a finite dimensional Lie algebra $h$, does not require the semisimplicity of $g$. Again the closure of $h$ does not require the finite dimensionality of $g$.

Proof. The proof proceeds by substitution of (1.2) into (1.1) followed by a solution of the resulting equations for the $a_{r}(k)$. However before doing this a few preliminaries are necessary. Let us first suppose we are given a set of functions $e_{t}\left(k, k^{\prime}\right): t=1,2 \ldots m$ for which

$$
\sum_{t=1}^{m} e_{t}\left(k, k^{\prime}\right) V_{\gamma}^{t}=0 \text { for all } \gamma \text {. }
$$

Then if the $e_{t}\left(k, k^{\prime}\right)$ do not all vanish identically (say $e_{s} \neq 0$ ) we can use (2.4) to eliminate $V_{\gamma}^{s}: \gamma=1,2 \ldots \mu$ from (1.2). Hence we can assume without loss of generality that the $e_{t}$ vanish identically. Combining this with the semisimplicity of $g$ (it is sufficient that $g$ coincide with its derived algebra) we have the implication

$$
\sum_{t=1}^{m} e_{t}\left(k, k^{\prime}\right) c_{\alpha \beta}^{\gamma} V_{\gamma}^{t}=0 \quad \text { for all } \alpha, \beta \Rightarrow e_{t}\left(k, k^{\prime}\right)=0 \text { for all } t .
$$

Again we may assume that the $a_{r}(k)$ are linearly independent. Then a simple induction argument shows that we may pick a set of points $k_{s}$ : $s=1,2 \ldots m$ in $R$ such that $\operatorname{det} a_{r}\left(k_{s}\right) \neq 0$. With this choice we set

where

$$
a_{r}^{\prime}(k)=\operatorname{det} a_{r s}(k) / \operatorname{det} a_{r}\left(k_{s}\right)
$$

$$
\begin{aligned}
a_{r s}(k) & =a_{r}(k): & & r=s \\
& =a_{r}\left(k_{s}\right): & & r \neq s .
\end{aligned}
$$

These quantities satisfy

$$
a_{r}{ }^{\prime}\left(k_{s}\right)=\delta_{r s}
$$

where $\delta_{r s}$ denotes the Kronecker delta. It is also clear that (2.6) may be solved for the $a_{i}(k)$ in terms of the $a_{i}^{\prime}(k)$ and so we may rewrite (1.2) in terms of the primed variables. This we do, dropping the prime. Then on substituting (1.2) into (1.1) which gives

$$
\sum_{r=1}^{m} \sum_{s=1}^{m} a_{r}(k) a_{s}\left(k^{\prime}\right)\left[V_{\alpha}^{r}, V_{\beta}^{s}\right]=c_{\alpha \beta}^{\gamma} \sum_{t=1}^{m} a_{t}\left(k+k^{\prime}\right) V_{\gamma}^{t}
$$


and using (2.7) we obtain

$$
\left[V_{\alpha}^{r}, V_{\beta}^{s}\right]=\sum_{t=1}^{m} c_{\alpha \beta}^{\gamma} a_{t}\left(k_{r}+k_{s}\right) V_{\gamma}^{t}
$$

This demonstrates the first part of the theorem. Observe that we have not so far used semisimplicity or finite dimensionality of $g$. Eq. (2.9) is not a complete statement of the commutation relations in $h$ since the $a_{t}(k)$ are not arbitrary. To determine these functions we substitute (2.9) back into (2.8) using (2.5). This shows that the $a_{r}(k)$ satisfy a relation of the form

$$
\sum_{r=1}^{m} \sum_{s=1}^{m} \beta_{r s t} a_{r}(k) a_{s}\left(k^{\prime}\right)=a_{t}\left(k+k^{\prime}\right)
$$

where the $\beta_{\text {rst }}$ are arbitrary constants. This is a matrix generalisation of Cauchy's equation (A, pp. 197, 353). Such equations may be solved by a variety of methods, the simplest of which and the one we shall adopt here being differentiation. Before we can do this we must show that the assumed measurability $(2.1)$ of the $a_{r}(k)$ implies their differentiability to all orders. The method used is standard (A, p. 190) and so we omit the details except to mention that we use (2.7) to impose a condition on the $\beta_{r s t}$ which is necessary for the proof.

Differentiating (2.10) $l$-times with respect to $k$, setting

$$
\frac{d^{l}}{d k^{l}} a_{r}(k)=b_{r}^{l}(k) \text { and } k=0, k^{\prime}=k,
$$

we obtain

$$
\sum_{r=1}^{m} \sum_{s=1}^{m} \beta_{r s t} b_{r}^{l}(0) b_{s}^{0}(k)=b_{t}^{l}(k) \text {. }
$$

The $(m+1)$-vectors $\boldsymbol{b}^{l}: l=0,1,2 \ldots m$ with components $b_{r}{ }^{l}(0)$ are necessarily linearly dependent. Hence there exist constants $\alpha_{l}$ : $l=0,1,2 \ldots m$ which are not all zero such that

$$
\sum_{l=0}^{m} \alpha_{l} \boldsymbol{b}^{l}=\mathbf{0} \quad \text { or } \quad \sum_{l=0}^{m} \alpha_{l} b_{r}{ }^{l}(0)=0 \text { for all } r .
$$

Multiplying both sides of (2.11) by $\alpha_{l}$ and summing over $l$, this then gives

$$
\sum_{l=0}^{m} \alpha_{l} \frac{d^{l}}{d k^{l}} a_{t}(k)=0
$$

for all $t$. We solve this differential equation for the $a_{r}(k)$. In this the following two distinct situations can arise. 
Case 1. The roots $\xi_{1}, \xi_{2}, \ldots \xi_{m}$ of the polynomial equation $\sum_{l=0}^{m} \alpha_{l} \xi^{l}=0$ are all distinct. Then by a suitable redefinition of the $V_{\alpha}^{r}$ we may set

$$
a_{r}(k)=\exp \xi_{r} k
$$

for all $r$. Substituting (2.13) back into (2.8) using the linear independence of the exponential function for different arguments, we obtain

$$
\left[V_{\alpha}^{r}, V_{\beta}^{s}\right]=\delta_{r s} c_{\alpha \beta}^{\gamma} V_{\gamma}^{r}
$$

which is just the $m$-fold direct sum of $g$.

Case 2. All roots equal. Then by a suitable redefinition of the $V_{\alpha}^{r}$ we may set

$$
a_{r}(k)=k^{r-1} \exp \xi k .
$$

Substitution of this into (2.8) gives (2.2) with $m_{i}=m$. The fact that (2.15) is a limiting form of the solution (2.13) should make the stated contraction property of (2.2) clear. To show this explicitly we let $\varepsilon$ be a positive number and set

$$
\xi_{r}=\xi+(r-1) \varepsilon, \quad a_{r}^{\prime}(k)=\left(\frac{1}{\varepsilon} \Delta_{\varepsilon}\right)^{r-1} \exp \xi k
$$

where $\Delta_{\varepsilon}$ is the difference operator which acts on the function $f$ according to

$$
\left(\Delta_{\varepsilon} f\right)(x)=f(x+\varepsilon)-f(x) .
$$

From (2.16) the primed variables $a_{r}^{\prime}(k)$ can be shown to take the form

$$
a_{r}^{\prime}(k)=\frac{1}{\varepsilon^{r-1}} \sum_{s=1}^{r}(-1)^{r-s}\left(\begin{array}{l}
r-1 \\
s-1
\end{array}\right) a_{s}(k)
$$

where the $a_{s}(k)$ are given by (2.13). By the property of $\Delta_{\varepsilon}$, this linear combination of the $a_{s}(k)$ become the quantities defined in $(2.15)$ in the limit $\varepsilon \rightarrow 0$. Correspondingly we define the primed elements $V_{\alpha}^{\prime}{ }^{r}$ through

$$
V_{\alpha} \equiv \sum_{r=1}^{m} a_{r}(k) V_{\alpha}^{r}=\sum_{r=1}^{m} a_{r}^{\prime}(k) V_{\alpha}^{\prime r}
$$

which gives, after a little reduction

$$
V_{\alpha}^{\prime r}=\varepsilon^{r-1} \sum_{s=r}^{m}\left(\begin{array}{l}
s-1 \\
r-1
\end{array}\right) V_{\alpha}^{s}
$$

Use of (2.17) and the known commutation relations (2.14) for the $V_{\alpha}^{s}$, we deduce

$$
\left[V_{\alpha}^{\prime r}, V_{\beta}^{\prime}{ }^{s}\right]=c_{\alpha \beta}^{\gamma} \sum_{t=(r, s)}^{r+s-1} \frac{(t-1) ! \varepsilon^{r+s-t-1}}{(r+s-t-1) !(t-r) !(t-s) !} V_{\gamma}^{\prime}{ }^{t}
$$


where we have used $(r, s)$ to denote the larger of $r$ and $s$. Taking the limit $\varepsilon \rightarrow 0$ in (2.18) we obtain (2.2) and this demonstrates the contraction.

Finally we remark that in the general case, when some roots are equal, $h$ is a direct sum of algebras $h_{i}$, each $h_{i}$ corresponding to a given root. The theorem is proved.

\section{Classification Theorem (General Case)}

We now state and prove the generalisation of Theorem 2.1 for the case when the $\boldsymbol{k}$-space is of arbitrary finite dimension. In this the measurability requirement $(2.1)$ on the $a_{r}(\boldsymbol{k})$ is assumed to hold in each coordinate separately at a fixed value of the remaining co-ordinates.

Theorem 3.1. Let $\mathscr{L} g$ be a local Lie algebra satisfying the requirements of Theorem 2.1 with $\boldsymbol{k} \in R^{n}$. Then $\mathscr{L} g$ satisfies the conclusions of Theorem 2.1 with each $h_{i}$ of the form

$$
\begin{aligned}
{\left[V_{\alpha}^{i_{1}, \ldots i_{n}}, V_{\beta}^{j_{1}, \ldots j_{n}}\right]=} & c_{\alpha \beta}^{\gamma}\left(\prod_{r=1}^{n} \frac{\left(i_{r}+j_{r}-2\right) !}{\left(i_{r}-1\right) !\left(j_{r}-1\right) !}\right) V_{\gamma}^{i_{1}+j_{1}-1 \ldots i_{n}+j_{n}-1}: \\
& 1 \leqq i_{r}+j_{r}-1 \leqq l_{r} \text { all } r \\
= & 0: \text { otherwise. }
\end{aligned}
$$

where the $l_{r}: r=1,2 \ldots n$ are positive integers and $i_{r}, j_{r}=1,2 \ldots l_{r}$ for all $r$. This is a contraction of the $\left(\prod_{r=1}^{n} l_{r}\right)$-fold direct sum of $g$. In each $h_{i}, V_{\alpha}(\boldsymbol{k})$
is given by

$$
V_{\alpha}(\boldsymbol{k})=\sum_{i_{1}=1}^{j_{1}}, \ldots, \sum_{i_{n}=1}^{j_{n}}\left(\prod_{r=1}^{n} k_{r}^{i_{r}} \exp \boldsymbol{\xi} \cdot \boldsymbol{k}\right) V_{\alpha}^{i_{1}, \ldots i_{n}}
$$

where $\xi$ is an $n$-tuple of arbitrary constants.

Proof. Substituting (1.2) into (1.1) and following through the analysis of Theorem 2.1, we obtain the Cauchy equation

$$
\sum_{r=1}^{m} \sum_{s=1}^{m} \beta_{r s t} a_{r}(\boldsymbol{k}) a_{s}\left(\boldsymbol{k}^{\prime}\right)=a_{t}\left(\boldsymbol{k}+\boldsymbol{k}^{\prime}\right)
$$

where the $\beta_{r s t}$ are arbitrary constants. Arguing as before we obtain from (3.3) a set of differential equations of the form

$$
\sum_{l=0}^{m} \alpha_{l}^{i} \frac{d^{l}}{d k_{i}^{l}} a_{t}(\boldsymbol{k})=0
$$

for all $i=1,2 \ldots n$, the $\alpha_{l}^{i}$ being arbitrary constants. Let $\xi_{i, r_{i}}: r_{i}=1,2 \ldots m_{i}$; $i=1,2 \ldots n$, where the $m_{i}$ are integers satisfying $1 \leqq m_{i} \leqq m$, denote for 
each $i$ the distinct roots of the polynomial equation

$$
\sum_{l=0}^{m} \alpha_{l}^{i} \xi^{l}=0
$$

Let $\xi$ be an $n$-tuple with $i^{\text {th }}$ entry $\xi_{i, r_{i}}$. Then

Lemma 3.1. There are at most $m$ different $n$-tuples $\xi$ that occur in each solution of (3.3).

Proof. Pick a solution of (3.3) and let $u$ be the number of $\xi n$-tuples occurring in it. Then we may write

$$
a_{\boldsymbol{r}}(\boldsymbol{k})=\sum_{v=1}^{u} b_{r v}(\boldsymbol{k}) \exp \boldsymbol{\xi}_{v} \cdot \boldsymbol{k}
$$

where the $b_{r v}(\boldsymbol{k})$ are at most polynomials in the $k_{s}: s=1,2 \ldots n$. We may assume without loss of generality that the $b_{r v}$ are linearly independent in the sense that $\sum_{r=1}^{m} \alpha_{r} b_{r v}=0$ for all $v$, implies $\alpha_{r}=0$ for all $r$. Otherwise we may eliminate at least one of the $\xi n$-tuples from (3.5). We show that $u \leqq m$ by substitution of (3.5) into (3.3). Through the linear independence of the exponential function for different arguments this gives

$$
\sum_{r=1}^{m} \sum_{s=1}^{m} \beta_{r s t} b_{r v}(\boldsymbol{k}) b_{s w}\left(\boldsymbol{k}^{\prime}\right)=0
$$

for all $t$ whenever $\boldsymbol{\xi}_{v} \neq \boldsymbol{\xi}_{w}$ and hence whenever $v \neq w$. If $u>m$, then through the linear independence of the $b_{r v}, \beta_{r s t}=0$ for all $r, s, t$, which is impossible. Hence $u \leqq m$ and the lemma is proved.

In virtue of the above lemma we may, in any given solution of (3.3), take a linear combination of the $V_{\alpha}^{r}$ such that the $a_{r}(\boldsymbol{k})$ each involve only one exponential function. It is clear that this gives the direct sum decomposition into the $h_{i}$. Thus it suffices to check the case when $u=1$ in (3.5), that is when

$$
a_{r}(\boldsymbol{k})=b_{\boldsymbol{r}}(\boldsymbol{k}) \exp \boldsymbol{\xi} \cdot \boldsymbol{k}
$$

for all $r=1,2 \ldots m$. Now the most general polynomial in the $k_{i}$ can always be written in the form

$$
\sum_{i_{1}=1}^{j_{1}} \ldots \sum_{i_{n}=1}^{j_{n}} \beta_{i_{1} \ldots i_{n}}\left(\prod_{r=1}^{n} k_{r}^{i_{r}}\right)
$$

where the $j_{r}: r=1,2 \ldots n$ are positive integers and the $\beta_{i_{1}, i_{2} \ldots i_{n}}$ are arbitrary constants. Setting each $b_{r}(\boldsymbol{k})$ equal to such an expression and substituting back into (3.6) and (1.2) we obtain (3.2) as required. Eq. (3.1) follows by equating coefficients in powers of the $k_{i}$. The contraction property follows through similar reasoning to that given in $\S 2$ and hence the theorem is proved. 
It is important to realise that the $V_{\alpha}^{i_{1}, i_{2}, \ldots i_{n}}$ defined above need not all be non-zero or linearly independent. Indeed for $n>1$, the general solution of (1.1) is prescribed only by including in (3.2) all linear relations of the form

$$
\sum_{i_{1}=1}^{j_{1}} \ldots \sum_{i_{n}=1}^{j_{n}} \gamma_{i_{1}, \ldots i_{n}} V_{\alpha}^{i_{1} \ldots i_{n}}=0
$$

which are consistent with the commutation relations given in (3.1). Finding all such linear relations is equivalent to determining all the polynomial solutions to (3.3), that is finding all the relations which hold between the $\beta_{i_{1} \ldots i_{n}}$ defined in (3.7). Actually in so far as a representation theory is concerned these additional constraints are unimportant since they will be automatically included as a set of conditions on the representation itself.

In analysing relations of the form (3.8), we may assume without loss of generality that $g$ is simple. This is because a semisimple Lie algebra can be expressed as a direct sum of its simple ideals [6] and this decomposition effects a separation of the local Lie algebra $\mathscr{L} g$ into mutually independent parts which we may consider separately. The following theorem describes all possible relations of the form given in (3.8).

Theorem 3.2. Let $\mathscr{L} g$ be a local Lie algebra satisfying the requirements of Theorem 3.1 with $g$ simple. Let $h_{i}$ be a Lie algebra defined through (3.1) which satisfies the conclusions. Suppose that (3.8) holds for some $\alpha$ and given constants $\gamma_{i_{1} \ldots i_{n}}$. Then it holds for all $\alpha$ and furthermore

$$
\sum_{i_{1}=1}^{j_{1}} \ldots \sum_{i_{n}=1}^{j_{n}} \gamma_{i_{1} \ldots i_{n}}\left(i_{s}+1\right) V_{\alpha}^{i_{1}, \ldots i_{s}+1, \ldots i_{n}}=0
$$

holds for all $s$ and for all $\alpha$. The set of all such relations are consistent with (3.1) and define the most general admissable $h_{i}$ appearing in $\mathscr{L} g$.

Proof. Using the simplicity of $g$ we first prove:

Lemma 3.2. Given any set of quantities $X_{\alpha}: \alpha=1,2 \ldots \mu$ such that for at least one value of $\alpha$ and for each positive integer $l$

$$
\begin{aligned}
X_{\alpha} & =0 \\
c_{\alpha \beta_{1}}^{\gamma_{1}} c_{\gamma_{1} \beta_{2}}^{\gamma_{2}} \ldots c_{\gamma_{l-1} \beta_{l}}^{\gamma_{l}} X_{\gamma_{l}} & =0
\end{aligned}
$$

for all $\beta_{1}, \ldots \beta_{l}$. Then

for all $\alpha$.

$$
X_{\alpha}=0
$$

Proof. Let $\bar{X}_{\alpha}: \alpha=1,2 \ldots \mu$ close on $g$. Then the set of relations (3.10) with $X$ replaced by $\bar{X}$ define a non-zero ideal in $g$ which by simplicity equals $g$. Hence these relations imply $\bar{X}_{\alpha}=0$ for all $\alpha$. Correspondingly (3.10) must imply (3.11). 
Let us use $V_{\alpha}^{0}$ to denote $V_{\alpha}^{00, \ldots 0}$ and $V_{\alpha}^{s}$ to denote $V_{\alpha}^{i_{1}, \ldots i_{n}}: i_{r}=\delta_{r s}$. Then through the above lemma if $V_{\alpha}^{0}=0$ for some $\alpha$, then $V_{\alpha}^{0}=0$ for all $\alpha$. Again by commuting $V_{\beta}^{0}: \beta=1,2 \ldots \mu$ through (3.8) we can show that the latter holds for all $\alpha$ if it holds for any one value. Then commuting $V_{\beta}^{s}$ through (3.8) and using (3.1) we obtain

$$
c_{\alpha \beta}^{\gamma}\left\{\sum_{i_{1}=1}^{j_{1}} \ldots \sum_{i_{n}=1}^{j_{n}} \gamma_{i_{1} \ldots i_{n}}\left(i_{s}+1\right) V_{\gamma}^{i_{1} \ldots i_{s}+1, \ldots i_{n}}\right\}=0
$$

for all $\alpha, \beta$. From this (3.9) follows by simplicity of $g$. The set of all relations of the form (3.9) is consistent with all Lie bracket relations (3.1) in which one of the factors is $V_{\alpha}^{s}: s=0,1, \ldots m$. By (3.1) these elements generate $h_{i}$ and so through the Jacobi identities the commutation relations (3.1) are consistent for the whole of $h_{i}$ and the theorem is proved.

As an illustration of Theorem 3.2, consider the simplest linear relation of the form (3.8). This occurs when only one of the $\gamma_{i_{1}} \ldots i_{n}$ are non-zero. Then

$$
V_{\alpha}^{i_{1}, \ldots i_{n}}=0
$$

which by the simplicity of $g$ holds for all $\alpha$. Theorem 3.2 states that

$$
V_{\alpha}^{l_{1}, \ldots l_{n}}=0
$$

must hold for all $\alpha$ and for those values of $l_{r}$ satisfying $l_{r} \geqq i_{r}$ for all $r=1,2, \ldots n$. This result can also be derived by studying the polynomial solutions to (3.3). Indeed it is clear that (3.13) implies that the monomial $\prod_{r=1}^{n} k_{r}{ }^{i_{r}}$ drops out of the summation in (3.2) and hence is not present in any of the $b_{r}(\boldsymbol{k})$. However if this holds then the monomials $\prod_{r=1}^{n} k_{r}^{l_{r}}$ : $l_{r} \geqq i_{r}$ for all $r$, must also be absent otherwise we obtain a contradiction through substitution in the right hand side of (3.3) and identification of coefficients in powers of the $k_{i}: i=1,2 \ldots n$. Through (3.2) the absence of these polynomials leads to (3.14) as we wished to show. This argument can be extended to give an alternative proof of Theorem 3.2.

The importance of Theorem 3.1 is that it provides an immediate classification of all possible continuous finite dimensional representations of a given local Lie algebra $\mathscr{L} g$ (with $g$ semisimple). In this we define the topology in $\mathscr{L} g$ through the distance function $\left|V_{\alpha}(\boldsymbol{k})-V_{\alpha}\left(\boldsymbol{k}^{\prime}\right)\right|$ which is assumed to satisfy the usual inequalities with respect to addition and multiplication of the $V_{\alpha}(\boldsymbol{k})$, the Lie bracket being represented by the commutator. By the translation property implicit in (1.1), $V_{\alpha}(\boldsymbol{k})$ is continuous over the whole of $R^{n}$ if it is continuous at any one point and we assume that this holds in the following. In virtue of the finite dimensionality of the representation the choice of (locally convex) topology in the representation space is immaterial [7]. It is convenient to choose 
the weak operator topology (Ref. [7], p. 441). The following theorem characterises the representations.

Theorem 3.3. Let $\mathscr{L} g$ be a local Lie algebra based on a finite dimensional semisimple Lie algebra of dimension $\mu$, with generators $V_{\alpha}(\boldsymbol{k})$ : $k \in R^{n}$; continuous over $R^{n}$. Then every continuous finite dimensional representation of $\mathscr{L} g$ has a direct sum decomposition into pieces of the form given in (3.2) where the $V_{\alpha}^{i_{1}, \ldots i_{n}}$ define a finite dimensional representation of (3.1).

Proof. Suppose the representation is of dimension $m$. Then there exists a basis $V_{r}: r=1,2 \ldots m^{2}$ in the representation space such that

$$
V_{\alpha}(\boldsymbol{k})=\sum_{i=1}^{m^{2}} a_{\alpha i}(\boldsymbol{k}) V_{i}
$$

holds for all $\alpha$ where the $a_{\alpha r}(\boldsymbol{k})$ are real or complex valued functions on $R^{n}$. Through the definition of the weak operator topology, continuity in the $V_{\alpha}(\boldsymbol{k})$ implies that the matrix elements $a_{\alpha i}(\boldsymbol{k})$ are continuous and hence measurable over any finite interval. It remains to show that (3.15) can be written in the form given in (1.2). This follows through the finite dimensionality of $g$ and of the representation which permits the relabelling

$$
a_{\alpha i}(\boldsymbol{k})=\sum_{r=1}^{m \mu} \beta_{\alpha i r} a_{r}(\boldsymbol{k})
$$

in which the $\beta_{\text {xir }}$ are constants. Substituting (3.16) into (3.15) we obtain (1.2) and the theorem is proved.

\section{Non-Semisimplicity of the Base Algebra}

The requirement that the base algebra $g$ be semisimple is essentially unnecessary, though omitting this condition does alter slightly the conclusions of Theorems 2.1 and 3.1. For example if $g$ is commutative, it follows from (2.9) that $h$ as defined above is also commutative as we might expect, whereas the functions $a_{r}(\boldsymbol{k})$ are arbitrary.

In general let $g^{\prime}$ denote the derived algebra of $g$ (Ref. [6], p. 10) and let $\left\{V_{\alpha}, W_{\beta}: \alpha=1,2 \ldots \mu ; \beta=1,2 \ldots v\right\}$ be a basis for $g$ such that $\left\{V_{\alpha}: \alpha=1,2 \ldots \mu\right\}$ is a basis for $g^{\prime}$. We shall assume for simplicity that the $k$-space is one dimensional. Then if (1.2) holds there exist integers $m, l$ with $m \geqq l$ such that

$$
V_{\alpha}=\sum_{r=1}^{l} a_{r}(k) V_{\alpha}^{r} ; \quad W_{\beta}=\sum_{r=1}^{m} a_{r}(k) W_{\beta}^{r}
$$

where the $a_{r}(k)$ are linearly independent and the $V_{\alpha}^{r}$ satisfy the implication

$$
\sum_{r=1}^{l} e_{r} V_{\alpha}^{r}=0 \quad \text { all } \quad \alpha \Rightarrow e_{r}=0 \quad \text { all } r
$$

9 Commun. math. Phys., Vol. 19 
(This is not imposed on the $W_{\alpha}^{r}$.) Following through the analysis of Theorem 2.1 we obtain, successively using the linear independence of the $a_{r}(k)$ and (4.2), that

$$
\sum_{r=1}^{l} \sum_{s=1}^{m} \beta_{r s t} a_{r}(k) a_{s}\left(k^{\prime}\right)=a_{t}\left(k+k^{\prime}\right)
$$

for all $t=1,2 \ldots l$; where the $\beta_{r s t}$ are constants. Further reduction gives the set of differential equations

$$
\sum_{s=0}^{l} \alpha_{s} \frac{d^{s}}{d k^{s}} a_{t}(k)=0
$$

for $t=1,2 \ldots l$. When all the roots $\xi_{1}, \xi_{2} \ldots \xi_{l}$ of the associated polynomial equation are distinct, we may write

$$
a_{r}(k)=\exp \xi_{r} k
$$

for all $r=1,2 \ldots l$. Substituting this back into (4.3) and using the linear independence of the exponential function for different arguments we obtain

$$
\sum_{s=1}^{m}\left(\beta_{r s t}-\delta_{r t}\right) a_{s}\left(k^{\prime}\right)=0
$$

for all $r, t=1,2 \ldots l$. Through the linear independence of the $a_{s}\left(k^{\prime}\right)$ this implies

$$
\beta_{r s t}=\delta_{r s} \delta_{r t}
$$

for all $r, t=1,2 \ldots l ; s=1,2 \ldots m$; which leads to a conclusion analogous to (2.14). More generally each distinct root defines a separate piece of $h$ which therefore has the direct sum decomposition as described in Theorem 2.1. Thus it is sufficient to consider the case when all roots are the same. We may then set

$$
a_{r}(k)=k^{r-1} \exp \xi k .
$$

Substituting (4.4) into (4.3), differentiating $u$ times with respect to $k$ and setting $k=0$, we obtain

$$
\sum_{s=1}^{m}\left\{\frac{(r-1) !}{(r-u) !} \beta_{u s t}-\frac{(t-1) !}{(t-u) !} \delta_{s t-u+1}\right\} a_{s}\left(k^{\prime}\right)=0
$$

for all $r, t, u=1,2 \ldots l$. Through the linear independence of the $a_{s}\left(k^{\prime}\right)$ this gives an explicit expression for the $\beta_{r s t}$, which after a little reduction results in the following commutation relation for the $V_{\alpha}^{r}, W_{\beta}^{s}$; namely

$$
\begin{array}{rlrl}
{\left[V_{\alpha}^{r}, W_{\beta}^{s}\right]} & =c_{\alpha \beta}^{\gamma} V_{\gamma}^{r+s-1} \frac{(r+s-2) !}{(r-1) !(s-1) !}: & r+s-1 \leqq l \\
& =0 \quad: \quad r+s-1>l
\end{array}
$$

for all $r=1,2 \ldots l ; s=1,2 \ldots m ; \alpha=1,2 \ldots \mu ; \beta=1,2 \ldots v$. 
This is essentially the same result as that obtained in the case when $g$ is semisimple. It differs in only two respects. Firstly the functions $a_{r}(k)$ : $r=l+1, l+2, \ldots m$ are arbitrary. Secondly the $V_{\alpha}^{r}$ are defined only for $r \leqq l$. The first difference is essentially trivial and does not effect the representation theory. As for the second we may formally define the $V_{\alpha}^{r}$ for $r>l$ assuming (4.5) to hold for all $r, s=1,2 \ldots m$, and then set these quantities equal to zero. This last step, which brings (2.2) into the form of (4.5) is not inconsistent with the extended commutation relations and so will be automatically included in the representation theory. We may sum up our conclusions as follows.

Theorem 4.1. Let $\mathscr{L} g$ be a local Lie algebra satisfying the conditions of Theorem 2.1 except that its base algebra $g$ is arbitrary. Let $g^{\prime}$ be the derived algebra of $g$ with $V_{\alpha}, W_{\beta}: \alpha=1,2 \ldots \mu ; \beta=1,2 \ldots v$ defined as above. Then $\mathscr{L} g$ satisfies the conclusions of Theorem 2.1 with each $h_{i}$ of the form given in (4.5). With respect to each $h_{i}, V_{\alpha}$ and $W_{\beta}$ are given by

$$
V_{\alpha}=\sum_{r=1}^{l} k^{r-1}(\exp \xi k) V_{\alpha}^{r}: W_{\beta}=\sum_{r=1}^{l} k^{r-1}(\exp \xi k) W_{\beta}^{r}+\sum_{s=l+1}^{m} a_{s}(k) W_{\beta}^{s}
$$

where $\xi$ is an arbitrary constant and the $a_{s}(k): s=l+1, l+2 \ldots m$ are arbitrary linearly independent functions linearly independent of the $k^{r-1} \exp \xi k: r=1,2 \ldots l$.

Remark. If $g$ is infinite, the same conclusions hold except that either $\mu$ or $v$ must be infinite and hence so are each $h_{i}$.

\section{Discussion}

In comparison with previous work on local Lie algebras we remark that Chang, Dashen and O'Raifeartaigh [4] have stated conclusions similar to ours - or at least in the same spirit. However these authors gave no proofs nor stated precise conditions under which their assertions held. There are also a number of omissions in their results. In particular they do not give the full set of commutation relations appearing in (3.1) or discuss the possible sets of relationships between the generators described in Theorem 3.2. Roffman [8] has derived all the finite dimensional representations of (1.1) (for $g$ semisimple) under conditions of irreducibility and skew symmetry of the representation. This work has been extended by Havlícek [9] to irreducible skew-symmetric representations of a related (discrete) local Lie algebra satisfying a condition similar to (1.2). Our results are more general than these in that we do not impose either skew-symmetry or irreducibility of the representation. Moreover our proof is direct, elementary and rigorous. We have also stated and shown explicitly certain contraction properties of these algebras and extended our results to arbitrary base algebra $g$. 
In a different spirit Mendes and Ne'eman [10] have studied representations of (1.1) with $g=s u(2)$ by an inducing procedure on the "isospin" subalgebra obtained by setting $\boldsymbol{k}=\mathbf{0}$ in (1.1). They derive all irreducible unitary representations having arbitrary isospin with multiplicity one. Though it is not a priori obvious, it turns out that all these representations are included in our classification. Indeed they correspond to representations of $s u(2) \times s u(2)$ and its contracted form $E(3)$ which is the case described by setting $m=2$ in Theorem 2.1 .

Though our analysis is independent of whether $\mathscr{L} g$ is over the real or complex field, it is worth mentioning that the complexification $h^{*}$ of the Lie algebra $h$ which appears in the decomposition of $\mathscr{L} g$ occurs in two stages. The first is through the choice of $g$ over the complex field, the second through the functions $a_{r}(\boldsymbol{k})$. Both steps are necessary and correspondingly to obtain all possible real forms of $h^{*}$ we must start from all possible real forms of $g^{*}$ and allow all possible real and imaginary multiples of the functions $a_{r}(k)$. Thus for $g=s o(3)$ and $m=2$, the uncontracted forms of $h$ are $s o(4)$ and $s o(3,1)$; whereas with $g=s o(2,1)$, they are $s o(2,2)$ and $s o(1,3)$. The hermiticity condition which here takes the form $V^{\dagger}(\boldsymbol{k})=-V(-\boldsymbol{k})$ requires the $a_{\boldsymbol{r}}(\boldsymbol{k})$ to be real multiples of the expressions given in $\$ \S 2,3$ and this excludes some of the possible real forms of $h^{*}$. In the above example only $s o(4)$ and $s o(2,2)$ remain.

The classification that we have given certainly does not embrace all possible representations of local Lie algebras, though it may well include all those useful in physics. It remains to be seen whether the relaxing of condition (1.2) can lead to unexpected and interesting results.

Acknowledgements. This work was supported by a Royal Society-Israel Academy Research Fellowship held at the Physics department of Tel-Aviv University. I should like to thank Professor Y. Ne'eman for suggesting the problem and for his enthusiastic interest in its development. Dr. M. Pavković also contributed some interesting discussion.

\section{References}

1. Gell-Mann, M.: Phys. Rev. 125, 1067 (1962).

2. Dashen, R. F., Gell-Mann, M.: Phys. Rev. Letters 17, 340 (1966).

3. - Sharp, D. H. S.: Phys. Rev. 165, 1857 (1968).

4. Chang, S. J., Dashen, R. F., O'Raifeartaigh, L.: Phys. Rev. 182, 1805 (1969).

5. Aczél, J.: Lectures on functional equations and their application. New York: Academic Press 1966.

6. Jacobson, N.: Lie algebras, p. 71. New York: Interscience 1962.

7. Naimark, M. A.: Normed rings, p. 51. Groningen: P. Noordhoff 1964.

8. Roffman, E. H.: J. Math. Phys. 8, 1954 (1967).

9. Havlíček, M.: Commun. Math. Phys. 13, 73 (1969).

10. Mendes, R. V., Ne'eman, Y.: J. Math. Phys. (in the press).

A. Joseph

Physics Department, Tel-Aviv University, Tel Aviv, Israel 